\title{
Power-Law Corrections to the Kubo Formula Vanish in Quantum Hall Systems
}

\author{
M. Klein and R. Seiler \\ Technische Universität Berlin, Fachbereich Mathematik MA 7-2, D-1000 Berlin 12
}

\begin{abstract}
In first order perturbation theory conductivity is given by the Kubo formula, which in a Quantum Hall System equals the first Chern class of a vector bundle. We apply the adiabatic theorem to show that these topological constraints quantize the averaged conductivity to all orders of perturbation theory. Hence the Kubo formula is valid to all orders.
\end{abstract}

\section{Introduction}

Integrality of conductivity in Quantum Hall Systems has its origin in the topology of its underlying parameter space: the two torus. This fact has emerged from the work of Laughlin [L] and of Thouless, Kohomoto, Nightingale, and de Nijs $\left[\mathrm{TKN}^{2}\right]$ and became clear in the work of Thouless and Niu [TN 1, TN 2], and Avron and Seiler [AS]; there it was shown that the Kubo formula for the Hall conductivity is the first Chern number of a line bundle over the torus.

The topological nature of Kubo's formula for the Hall conductivity is very satisfactory because of the astounding experimental precision of the phenomenon. However the question of the validity of the Kubo formula and the corrections to Ohm's law had to be tackled in order to give a satisfactory picture. For that reason charge transport or- what is the same - the time averaged conductivity was analyzed in [ASY]. It was shown that - provided the gap condition holds conductivity is given by Kubo's formula and that the correction terms are at least quadratic in the electric potential $V$. It is the purpose of this article to prove the stronger statement that the corrections to Ohm's law are of order $V^{\infty}$.

We shall now give a short review of the model, methods and results. For technical details we refer to the later sections. The model is basically the same as in [AS]. This time however we use a rectangular configuration space and not the two hole geometry. This is irrelevant for all major results. Interaction with impurities and among electrons is included. For concreteness we choose Coulomb forces although the specific form of the electron interaction is irrelevant. The electric field is generated by a vector potential proportional to $\phi_{1}$. As in [ASY] it is switched on 
and off smoothly and adiabatically over a time scale $\tau, \phi_{1}=f(s=t / \tau) ; t$ denotes physical time, $s$ scaled time and $f$ the switching function. The current operator is defined in terms of its conjugate variable $\phi_{2}$. Dynamics is given through the multiparticle Hamiltonian $\widetilde{H}(\phi)$ respectively $\hat{H}(\phi), \phi \in \mathbb{R}^{2} ; \hat{H}(\phi)$ is doubly periodic in $\phi_{1}$ and $\phi_{2}$ by construction.

The gap condition can now be formulated. It says that the spectrum of $\hat{H}(\phi)$ splits into two disconnected pieces, separated by a gap. The spectral projector on the lower part is denoted by $\hat{P}(\phi)$ and is assumed to have finite dimension $q$. Hence $\hat{P}(\phi)$ defines a vector bundle $\widehat{B}$ over the two torus.

The main result, stated in Theorem 4.1, concerns the charge transport $Q$ defined in terms of the Hall-current $I$

$$
Q(\Phi)=\tau \int d s I(s, \Phi), \quad \Phi:=\phi_{2} .
$$

The $\Phi$ average of the transported charge $Q$ satisfies the equation

$$
\langle Q\rangle=\int_{0}^{2 \pi} \frac{d \Phi}{2 \pi} Q(\Phi)=\frac{i}{2 \pi q} \int_{T} \operatorname{Trace} \hat{P} d \hat{P} d \hat{P}+O\left(1 / \tau^{\infty}\right) .
$$

The first term on the right-hand side is an integer divided by the dimension $q$ because of its geometric significance. In fact $i$ Trace $\hat{P} d \hat{P} d \hat{P}$ is the first Chern class of the vector bundle $\widehat{B}$. Integrality of

$$
\frac{i}{2 \pi} \int_{T} \operatorname{Trace} \hat{P} d \hat{P} d \hat{P}
$$

follows from an elementary computation.

The above result can be reinterpreted in terms of the averaged Hall conductivity $\sigma$. For that we choose the switching function in such a way that $f^{\prime}(s)=2 \pi$ for all $s$ between switching on and off (hence the graph of $f$ consists of 3 linear pieces which are smoothly connected). So the Hall voltage during this time interval is $V=2 \pi / \tau$. The time and $\Phi$ averaged Hall current becomes now

$$
\begin{aligned}
\langle I\rangle & =\int_{0}^{1} d s \int_{0}^{2 \pi} \frac{d \Phi}{2 \pi} I(s, \Phi)=\sigma V+O\left(V^{\infty}\right), \\
\sigma & =\frac{i}{q(2 \pi)^{2}} \int_{T} \operatorname{Trace} \hat{P} d \hat{P} d \hat{P} .
\end{aligned}
$$

Hence $2 \pi \sigma$ is an integer.

In order to apply the above theoretical results to physical reality one has to discuss the following three questions:

1. Does the multiparticle Hamiltonian satisfy the gap condition?

2. Is the groundstate degenerate or nondegenerate?

3. What is the numerical value of the adiabatic parameter?

There are two points of view for looking at the question of a gap in the spectrum of $\hat{H}(\phi)$. The first one is very general and makes use of the von NeumannWigner theorem [ASS]. This theorem states that complex hermitian matrices with degenerate spectrum have real codimension three in the set of all hermitian matrices. Hence a two-parameter family of Hamiltonians has generically non- 
degenerate spectrum and in particular a gap. Nothing is said about the size of the gap. It could be very small compared to thermal energies.

The second point of view brings in the effective two-dimensionality of the experimental setup and makes use of a very crude one-particle approximation. In the experiment one has an electric field perpendicular to the sample plane that keeps the electrons in the vicinity of the insulating material surface (in within a few Angstroms). The electrons move therefore in a plane under the influence of a strong magnetic field of about 5 Tesla. The experimental parameters are arranged in such a way that the transversal excitation energies are several times larger than the Landau energies of a few meVolt. So in this very crude approximation the Hamiltonian has a gap if the Fermi energy lies between Landau levels.

Now we turn to the second question. In view of Eq. (1.4) the experimental result suggests that in the case of the ordinary QHE the physical state is nondegenerate. In the case of the fractional QHE one expects a degeneracy $q=1 / v$ of the physical state, where $v$ denotes the filling factor (the filling factor is the number of magnetic flux units through the sample divided by the number of electrons in the sample). This is in conflict with an analysis based on the von Neumann-Wigner theorem [ASS], where only generic Hamiltonians are considered. However there is good reason to believe that Hamiltonians modeling Quantum Hall Systems with fractional conductivities are non-generic: The fractional QHE is typically observed in very clean material. This suggests that one looks at a model Hamiltonian of the type $\tilde{H}(\phi)$ as defined in the next section (2.1) with all potentials $V$ set equal to zero because the $V$ 's stand for the interactions with impurities. This family of operators has - after a minor change of boundary conditions degenerate discrete spectrum. The degeneracy of each eigenvalue is a multiple of $1 / v$. Since all this holds for every $\phi \in \mathbb{R}^{2}$, the operator is manifestly non-generic.

Now we come to the third question: The definition and size of the adiabatic parameter. The multiparticle Hamiltonian $\tilde{H}(\phi)$ modeling the Quantum Hall System is defined in the next section. Here we need just its general structure. Furthermore we replace the atomic units by the conventional MKS units:

$$
\tilde{H}(\phi)=\frac{1}{2 m}\left(-\frac{\hbar}{i} \nabla-e A-\phi_{1} e a_{1}-\phi_{2} e a_{2}\right)^{2}+W .
$$

$W$ stands for all interactions among particles and of particles with impurities. $\tilde{H}$ is periodic in both variables up to a unitary transformation with period $h / e$. Now $\phi_{1}=-V t$, where $V$ denotes the electric potential and $t$ time. Hence $\tilde{H}\left(\phi_{1}=-V t, \phi_{2}\right)$ is periodic in time with period $\tau=h / e V, \tau$ is the adiabatic time scale. If we rewrite the Schrödinger equation

$$
i \hbar \partial_{t} \Psi=\tilde{H}\left(\phi_{1}=-V t, \phi_{2}\right) \Psi
$$

in terms of the scaled time $s, t=\tau s$, we get

$$
i \partial_{s} \Psi=\left(\frac{2 \pi}{e V}\right) H(s, \Phi) \Psi .
$$

Here we have defined the Hamiltonian $H$ by $H(s, \Phi)=\tilde{H}\left(\phi_{1}=-V \tau s, \phi_{2}=\Phi\right)$. By construction it is periodic in $s$ with period one and periodic in $\Phi$ with period $h / e$. 
The adiabatic expansion is generated by partial integration with respect to the scaled time using the Schrödinger equation (1.6) ([AS, ASY], see also the appendix for details). In every step one introduces therefore a term

$$
\alpha=\frac{e V}{2 \pi \Delta},
$$

where $\Delta$ denotes the gap in the spectrum of $H$. We call $\alpha$ the adiabatic parameter. It is a number proportional to the adiabatic time $\tau$. To get a rough estimate on $\alpha$ we set $\Delta$ equal to the Landau energy, $V=10 \mathrm{mV}, B=3$ Tesla and the effective mass of the electron $7 / 100$ electron masses. This gives for $\alpha$ the value $1 / 4$. In this approximation $\alpha$ is proportional to $V$ and $1 / B$.

The main tool in this article is the adiabatic theorem [K2, ASY]. It says that the physical time evolution $U_{\tau}$ defined by the initial value problem

$$
\begin{aligned}
i \partial_{s} U_{\tau}(s, \Phi) & =\tau H(s, \Phi) U_{\tau}(s, \Phi), \quad(s, \Phi) \in \mathbb{R}^{2}, \tau>0, \\
U_{\tau}(0, \Phi) & =1,
\end{aligned}
$$

is approximated in the limit $\tau \rightarrow \infty$ by the adiabatic evolution $U_{A}(s, \Phi)$ defined by

$$
\begin{aligned}
i \partial_{s} U_{A}(s, \Phi) & =\tau H_{A}(s, \Phi) U_{\tau}(s, \Phi), \\
U_{\tau}(0, \Phi) & =1, \\
H_{A}(s, \Phi) & =H(s, \Phi)+\frac{i}{\tau}\left[\partial_{s} P(s, \Phi), P(s, \Phi)\right] .
\end{aligned}
$$

There are two remarkable facts about $U_{A}$ :

1. Let $s_{0} \in \mathbb{R}$ be such that $H(s)$ is constant in the vicinity of $s_{0}$. Then $U_{\tau}$ approximates $U_{A}$ on the range of $P(0, \Phi)$ very well in the following sense:

$$
P(0, \Phi) U_{A}^{*}\left(s_{0}, \Phi\right) U_{\tau}\left(s_{0}, \Phi\right) P(0, \Phi)=0\left(1 / \tau^{\infty}\right), \quad \Phi \in \mathbb{R}^{2} .
$$

2. The adiabatic evolution has the property (intertwining property)

$$
U_{A}(s, \Phi) P(0, \Phi)=P(s, \Phi) U_{A}(s, \Phi), \quad(s, \Phi) \in \mathbb{R}^{2} .
$$

The intertwining property can be interpreted in a more geometric way leading to a better understanding of the main result (Theorem 4.1). Consider the vector bundle $B$ with base $\mathbb{R}^{2}$ and fiber range $P(s, \Phi),(s, \Phi) \in \mathbb{R}^{2}$. On $B$ we define the connection

$$
\nabla_{H}=d-[(d P), P]+i \tau H(d s+d \Phi) .
$$

By construction $U_{A}$ is a solution of the differential equation

$$
\left(X \nabla_{H}\right) U_{A}=0
$$

where $X$ denotes the vector field $(\partial / \partial s, 0)$ on $\mathbb{R}^{2}$. Hence $U_{A}$ is the integral of the connection $\nabla_{H}$ along $X$. The leading term in the adiabatic expansion of the averaged charge transport $\langle Q\rangle$ turns out to be the trace of the holonomy of $\nabla_{H}$ around the boundary of the rectangle $R=[0,1] \times[0,2 \pi]$, (Berry's Phase).

Now $H(s, \Phi)$ is up to unitaries periodic in $s$ and $\Phi$ with periods 1 and $2 \pi$. Hence one can substitute in leading order for $\langle Q\rangle$ the connection $\nabla_{H}$ by the connection 
$\hat{\nabla}=d-[(d \hat{P}), \hat{P}]$ on the vector bundle $\hat{B}$. The holonomy of $\nabla_{H}$ on $\hat{B}$ turns into a Chern number of $\hat{B}$ (Lemma 4.2).

To estimate the remainder term in the adiabatic expansion one proves that modulo terms of order $\tau^{-\infty}$ the rest is again a Chern number of a connection. Due to the adiabatic theorem it has to be small in $1 / \tau$. So it has to vanish.

To end this section let us mention that the connection $\hat{V}$ on $\hat{B}$ has a well known and natural origin. Consider the trivial Hilbert bundle $T \times \mathscr{H}$, where $T$ is the two torus and $\mathscr{H}$ denotes the space of many body states. The most trivial connection is the derivative with respect to the coordinates of $T$. Out of that the canonical connection $\nabla$ is constructed:

$$
\nabla:=\hat{P} d \hat{P}+(1-\hat{P}) d(1-\hat{P}) .
$$

This is done so as to respect the splitting of $T \times \mathscr{H}$ into $\widehat{B}$ and its orthogonal complement $\hat{B}^{\perp}$. The latter is defined in analogy to $\widehat{B}$ using the projectors $1-\hat{P}$. Now $\hat{\nabla}$ is the restriction of $\nabla$ to $\hat{B}$ :

$$
\hat{\nabla}=\hat{P} d .
$$

One finds by a straightforward computation for the corresponding curvature operator $\Omega$ the expression

$$
\widehat{\Omega}:=\hat{\nabla}^{2}=\hat{P}(d \hat{P})(d \hat{P}) \hat{P} .
$$

Hence the first Chern character $i / 2 \pi$ Trace $\hat{\Omega}$ is the expression given in formula 1.3.

This article is organized as follows: In the next section we introduce the model in detail. In Sect. 3 the Hall current and charge transport is discussed. A ChernSimons type formula turns out to be of great use. The fourth section contains the basic estimate on the corrections to the Kubo formula for charge transport and a short proof of integrality of the Chern numbers for the situation under discussion. To make the article selfcontained we collect in an appendix the main points about the adiabatic theorem as presented in reference [ASY]. This gives us at the same time the opportunity to correct a mistake in the original proof.

\section{The Model}

We now describe in detail the dynamics of a Quantum Hall System. It involves $N$ electrons moving in configuration space $A$ in an external magnetic field $B$ and an external electric field $E$ perpendicular to $B$ (Fig. 1). We are interested in the current perpendicular to $B$ and $E$, the Hall current $I$.

The configuration space is assumed to be the closed rectangle $A=\left[0, L_{1}\right]$ $\times\left[0, L_{2}\right]$ in $\mathbb{R}^{2}$. Other configurations are possible too, for instance the two hole geometry in 2 or 3 dimensions [ASY]. The external magnetic field $B$ is introduced through a (smooth) vector potential $A$ and gives rise to the velocity operator $v=p-A$.

The electric field is generated by a time dependent vector potential through the following construction: Letting $e_{1}$ be the unit vector in the 1-direction, $a_{1}=e_{1} / L_{1}$ is the vector potential which "generates a flux unit through loop 1" (Fig. 1). 


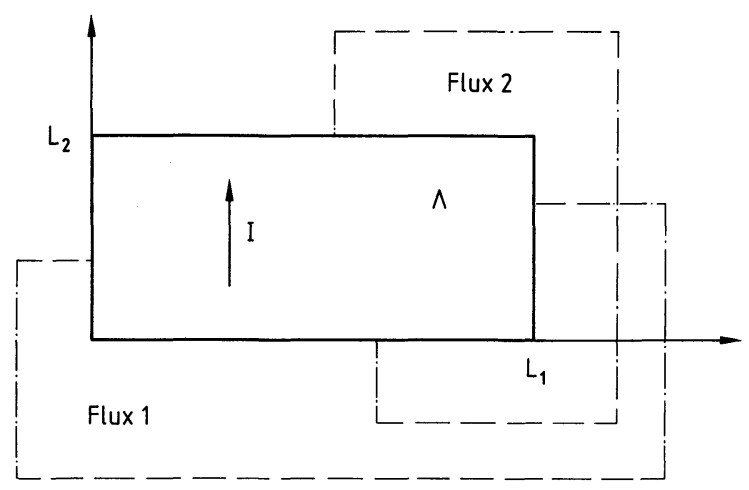

Fig. 1. Geometry of a quantum Hall system. The time dependent flux 1 through loop 1 induces the Hall current $I$ through loop 2

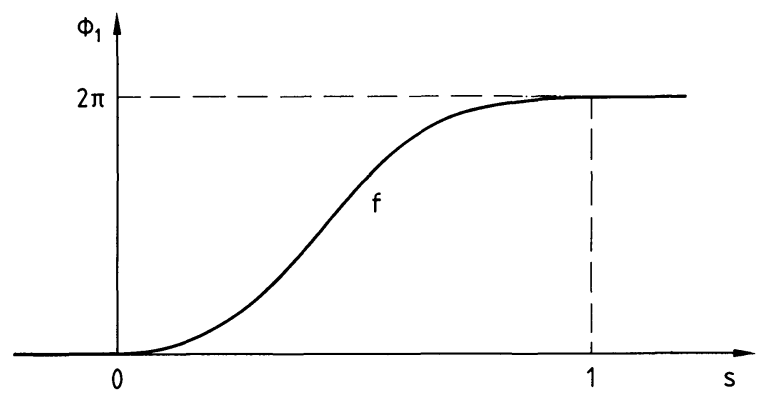

Fig. 2. The graph of the switching function

Furthermore, letting $t$ be time, $\tau$ the time scale, we take $f \in C^{\infty}(\mathbb{R}, \mathbb{R})$ to be a switching function of the scaled time $s=t / \tau$. It is supposed to grow monotonically from 0 to $2 \pi$ within $(0,1)$ (Fig. 2$)$. In particular, $\dot{f} \in C_{0}^{\infty}((0,1), \mathbb{R})$. By Faraday's law, the flux $\phi_{1}=f(s)$ through loop 1 gives rise to an electromotive force of size $1 / \tau \cdot f(s)$ across the rectangle $\Lambda$.

The observable of the Hall current $I$ is most easily defined by means of a second flux $\phi_{2}$ through loop 2 (Fig. 1) and a corresponding "unit vector potential" $a_{2}=e_{2} / L_{2}$, where $e_{2}$ denotes the unit vector in the 2-direction of $\mathbb{R}^{2}$. $\phi_{2}$ serves as a conjugate variable to define the current operator in formula (3.1) below.

The electrons interact among themselves by Coulomb repulsion and with impurities. The corresponding potential $V$ typically involves singularities. To cover this case in sufficient generality we consider for $\phi=\left(\phi_{1}, \phi_{2}\right) \in \mathbb{R}^{2}$ the Hamiltonian

$$
\widetilde{H}(\phi)=\sum_{i=1}^{N} \frac{1}{2}\left(v_{i}-\phi_{1} a_{1}-\phi_{2} a_{2}\right)^{2}+V\left(x_{i}\right)+\sum_{j<i} \frac{1}{\left|x_{i}-x_{j}\right|}
$$

with periodic boundary conditions on the antisymmetric (fermionic) part of $N$

$\otimes L^{2}(\Lambda)$ and assume 
H1: The potential $V$ is $-\Delta$-form bounded with relative bound zero, i.e.

$$
\langle\Psi, V \Psi\rangle \mid \leqq \varepsilon\|\nabla \Psi\|^{2}+C_{\varepsilon}\|\Psi\|^{2} \quad(\varepsilon>0)
$$

for all $\Psi \in H^{1}(\Lambda) . H^{1}(\Lambda)$ denotes the first Sobolev space on the rectangle $\Lambda$.

We remark that in dimension $n=2$ the Coulomb potential is not operator bounded with respect to $-\Delta$. Our assumption (H1), however, allows us to treat any finite superposition of Coulomb-like singularities in the sense of quadratic forms [K 1, p. 352].

To apply the adiabatic theorem to our situation we need some control on the operator domain of $H(\phi)$. This is given by the following technical

Lemma 2.1. Assume (H1). Then the family of operators $\tilde{H}(\phi)$ is type A analytic in $\phi \in \mathbb{R}^{2}$ with operator domain $D=D(\widetilde{H}(0))$ contained in the Sobolev space $H^{1}\left(\Lambda^{N}\right)$.

Proof. It follows from the positivity of $\frac{1}{\left|x_{i}-x_{j}\right|}$ and the form - boundedness of $V$
that

$$
t_{0}[\Psi]=\|\nabla \Psi\|^{2}+\left\langle\Psi, \sum_{i=1}^{N}\left(V\left(x_{i}\right)+\sum_{j<i} \frac{1}{\left|x_{i}-x_{j}\right|}\right) \Psi\right\rangle
$$

defines a closed symmetric semibounded form on

$$
Q\left(H_{0}\right)=\bigotimes_{i=1}^{N}\left\{\Psi \in H^{1}(\Lambda) ; \Psi(0, y)=\Psi\left(L^{1}, y\right), \Psi(x, 0)=\left(x, L_{2}\right)\right\},
$$

where the periodicity is understood in $L^{2}$-sense on the boundary of $\Lambda$.

According to the first representation theorem $[\mathrm{K} 1], t_{0}$ defines a unique selfadjoint operator $H_{0}$ in $L^{2}\left(\Lambda^{N}\right)$ with operator domain $D \subset Q\left(H_{0}\right)$. Furthermore, we have for all $\Psi \in D$,

$$
\begin{aligned}
\|\nabla \Psi\|^{2} & \leqq\left\langle\Psi, H_{0} \Psi\right\rangle-\left\langle\Psi, \sum_{i=1}^{N} V\left(x_{i}\right) \psi\right\rangle \\
& \leqq \delta\left\|H_{0} \Psi\right\|^{2}+\delta^{-1}\|\Psi\|^{2}+\varepsilon\|\nabla \Psi\|^{2}+C_{\varepsilon}\|\Psi\|^{2}
\end{aligned}
$$

for any $\varepsilon, \delta>0$, using Schwarz inequality and (2.2). This gives

$$
\|\nabla \Psi\|^{2}<\varepsilon\left\|H_{0} \Psi\right\|+C_{\varepsilon}\|\Psi\| \quad(\Psi \in D, \varepsilon>0) .
$$

Including the vector potential $A(x)+\phi_{1} a_{1}+\phi_{2} a_{2}$ in the definition of $\tilde{H}(\phi)$ induces a perturbation of $H_{0}$ which clearly is bounded as a map from $H^{1}\left(\Lambda^{N}\right)$ to $L^{2}\left(\Lambda^{N}\right)$. Thus $\widetilde{H}(\phi)$ is self-adjoint on $D$ for all $\phi \in \mathbb{R}^{2}$, and on that domain analyticity in $\phi$ is obvious in view of (2.5). Restricting $\tilde{H}(\phi)$ to the fermionic part of $\bigotimes_{j=1}^{N} L^{2}(\Lambda)$ proves
Lemma 2.1.

$\widetilde{H}(\phi)$ is up to unitary transformations periodic in both variables. To see this, we set for $i=1,2$,

$$
F_{i}(x)=\sum_{j=1}^{N}\left\langle a_{i}, x_{j}\right\rangle, \quad x=\left(x_{1}, \ldots, x_{N}\right) \in \Lambda^{N},
$$


and introduce the gauge transformation

$$
G(\phi)=e^{i\left(\phi_{1} F_{1}+\phi_{2} F_{2}\right)} .
$$

Then

$$
\hat{H}(\phi)=G(\phi) \tilde{H}(\phi) G^{*}(\phi)
$$

is $2 \pi$-periodic in $\phi_{1}$ and $\phi_{2}$, since its symbol is formally $\phi$-independent and its domain $D(\phi)=G(\phi) D$ is $2 \pi$-periodic by construction.

At this point we want to formulate the main hypothesis of this whole approach, the gap condition $[\mathrm{AS}]$ :

H2: The spectrum $\sigma(\hat{H}(\phi))$ has a gap (Fig. 3), i.e. there are continuous functions $g_{ \pm}: \mathbb{R}^{2} \rightarrow \mathbb{R}$ such that for some $\varepsilon>0$,

$$
\inf _{\phi \in \mathbb{R}^{2}} \operatorname{dist}\left(\left\{g_{-}(\phi), g_{+}(\phi)\right\}, \sigma(\hat{H}(\phi))\right)>\varepsilon .
$$

Note that due to the periodicity of $\hat{H}(\phi)$ and Eq. (2.8) we actually could restrict the values of $\phi$ to the square $[0,2 \pi]^{2}$ in $\mathbb{R}^{2}$.

One of the main consequences of hypothesis $(\mathrm{H} 2)$ is that the spectral projection of $\tilde{H}(\phi)$ or $\hat{H}(\phi)$ associated to the spectrum $\sigma(\tilde{H}(\phi))$ within the interval $\left(g_{-}(\phi), g_{+}(\phi)\right)$ is real analytic in $\phi$. This follows from Lemma 2.1 and the integral

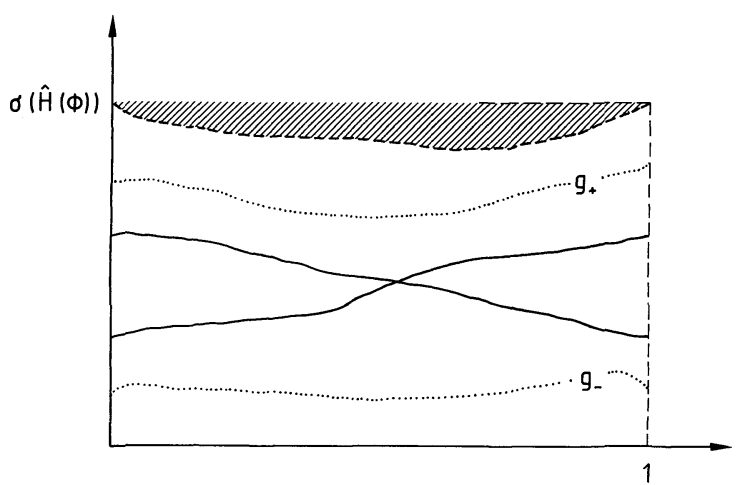

Fig. 3. The part of the spectrum of $\hat{H}(\Phi)$ within the interval $\left[g_{-}(\Phi), g_{+}(\Phi)\right]$ is separated by a gap from the rest of the spectrum

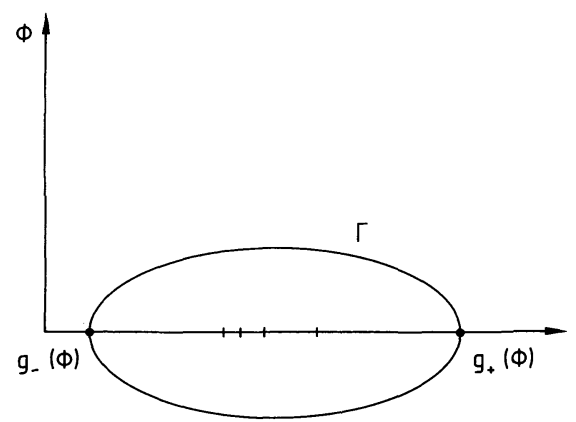

Fig. 4. The contour $\Gamma$ defining the projection $P(\Phi)$ 
representation

$$
\tilde{P}(\phi)=\frac{1}{2 \pi i} \int_{\Gamma(\phi)}(\tilde{H}(\phi)-z)^{-1} d z,
$$

where the complex contour $\Gamma(\phi)$ crosses the real axis at $g_{ \pm}(\phi)$ (Fig. 4).

The physical time evolution of our QHS is then given by the time dependent Hamiltonian

$$
H(s, \Phi)=\tilde{H}\left(\phi_{1}=f(s), \phi_{2}=\Phi\right) .
$$

For fixed $\Phi$, it satisfies the conditions (i-iii) of our Appendix. These ensure that the theorem of Kato and Yoshida about existence and uniqueness of the solution to the time dependent Schrödinger equation is applicable ([Y, $\mathrm{Kr}])$. We have

Lemma 2.2. For all $\Psi \in D$, the initial value problem

$$
i \partial_{s} U_{\tau}(s, \Phi) \Psi=\tau H(s, \Phi) U_{\tau}(s, \Phi) \Psi ; \quad U_{\tau}(0, \Phi) \Psi=\Psi
$$

possesses a unique solution which satisfies:

$U_{\tau}(s, \Phi)$ is unitary, strongly continuous in $s, \Phi \in \mathbb{R}$ and $U_{\tau}(s, \Phi)$ is continuously differentiable in $s, \Phi \in \mathbb{R}$ for all $\Psi \in D$. An analogous statement holds for the adiabatic propagator $U_{A}(s, \Phi)$.

For the proof of differentiability in $\Phi$ we refer to [ASY]. It is based upon Duhamels principle. Finally, we replace the pure state

$$
\Psi_{\tau}(s, \Phi)=U_{\tau}(s, \Phi) \Psi_{0}(\Phi)
$$

by the mixture

$$
P_{\tau}(s, \Phi)=U_{\tau}(s, \Phi) P(0, \Phi) U_{\tau}^{*}(s, \Phi),
$$

where $P(0, \Phi)$ is the spectral projection of $H(0, \Phi)$ associated to the spectrum of $H(0, \Phi)$ within the interval $\left(g_{-}(0, \Phi), g_{+}(0, \Phi)\right)$.

This slight generalization makes the following analysis much more transparent and allows us at the same time to say something about the fractional Quantum Hall effect. We remark that the state $P_{\tau}(s, \Phi)$ is equivalent to the pure state $\Psi_{\tau}(s, \Phi)$ if $P(0, \Phi)$ is the one-dimensional projection on $\Psi_{0}(\Phi) . P_{\tau}(s, \Phi)$ will be referred to as "the physical state."

\section{Hall Current and Charge Transport}

The Hall current $I$ is given in terms of the physical state $P_{\tau}$ and the Hamiltonian $H$ by

$$
I(s, \Phi)=\frac{1}{q} \operatorname{Tr} P_{\tau}(s, \Phi) \partial_{\Phi} H(s, \Phi) \quad(s, \Phi \in \mathbb{R}) .
$$

The above expression reduces to the formulae given earlier in [L, AS, ASY] if $q=\operatorname{dim} P(s, \Phi)=1$. The main result of this section is formula (3.2) below, which will be the point of departure for deriving the Kubo formula and estimating the remainder term in Sect. IV. 
Proposition 3.1. Letting $P_{\Phi}=P(0, \Phi)$, the Hall current $I$ can be represented as

$$
I=\frac{i}{q \tau} \partial_{s} \operatorname{Tr} P_{\Phi} U_{\tau}^{*} \partial_{\Phi} U_{\tau} .
$$

Proof. In view of Lemma 2.2, the derivatives in the above expression exist. Thus we get from (3.1), suppressing dependence on $s, \Phi$,

$$
q I=\operatorname{Tr} P_{\tau} \partial_{\Phi} H=\operatorname{Tr} P_{\Phi} U_{\tau}^{*}\left(\partial_{\Phi} H\right) U_{\tau}=\operatorname{Tr} P_{\Phi} U_{\tau}^{*} \partial_{\Phi}\left(H U_{\tau}\right)-\operatorname{Tr} P_{\Phi} U_{\tau}^{*} H \partial_{\Phi} U_{\tau} .
$$

Using the Schrödinger equation $i \partial_{s} U_{\tau}=\tau H U_{\tau}, H$ can be replaced by a time derivative. This gives

$$
I=\frac{i}{q \tau} \operatorname{Tr}\left\{P_{\Phi} U_{\tau}^{*} \partial_{\Phi} \partial_{s} U_{\tau}+P_{\Phi}\left(\partial_{s} U_{\tau}\right)^{*} \partial_{\Phi} U_{\tau}\right\},
$$

and proves the proposition.

For the interpretation of formula (3.2) we need the following version of the Chern-Simons formula:

Lemma 3.2. Let $P$ be a function on $\mathbb{R}^{n}$ with values in the projections of finite rank in some Hilbert space. Let $U$ be a function on $\mathbb{R}^{n}$ with values in the invertible linear operators on this Hilbert space. Assuming $P$ and $U$ to be (strongly) differentiable, the following identity holds:

$$
\operatorname{Tr} P_{u} d P_{u} d P_{u}=\operatorname{Tr} P d P d P+d\left(\operatorname{Tr} P U^{-1} d U\right),
$$

where we used the notation $P_{u}=U P U^{-1}$ and the product $d P d P$ is understood to be the wedge product of projection valued differential forms.

Proof. First note the following identity:

$$
d P_{u}=U([M, P]+d P) U^{-1},
$$

where we introduced the notation $M=U^{-1} d U$. It follows by direct computation from the definition of $P_{u}$. Formula (3.6) leads to

$$
P_{u} d P_{u} d P_{u}=U\left(K_{1}+K_{2}+P d P d P\right) U^{-1},
$$

where

$$
\begin{aligned}
& K_{1}=P[M, P][M, P], \\
& K_{2}=P[M, P] d P+P d P[M, P] .
\end{aligned}
$$

Computing term by term, we get

$$
\begin{aligned}
& K_{1}=P M P M P-P M M P, \\
& K_{2}=P M P d P-P M d P+P d P M P .
\end{aligned}
$$

We have used the trivial but important identity

$$
P d P P=0,
$$

which follows from

$$
d P=P d P+d P P
$$


The first terms of $K_{1}$ and $K_{2}$ do not contribute to the trace; hence

$$
\begin{aligned}
\operatorname{Tr} P_{u} d P_{u} d P_{u}-\operatorname{Tr} P d P d P & =-\operatorname{Tr} P M M P-\operatorname{Tr} P M d P+\operatorname{Tr} P d P M \\
& =-\operatorname{Tr} P M M P+\operatorname{Tr} d P M,
\end{aligned}
$$

where we used (3.10), cyclic invariance of the trace and anticommutativity of the differential forms $d P$ and $M$. Finally we use

$$
d M=d\left(U^{-1} d U\right)=-U^{-1} d U U^{-1} d U=-M M
$$

to get formula (3.5).

The basic formula (3.2) can be recast into another form which allows for an appealing interpretation. This is not necessary to derive our main result on the Kubo formula in Sect. IV. However, to clarify the geometric character of charge transport in a QHS, we state the following:

Proposition 3.3. Interpreting the Hall current as a differential 2-form, it is given by

where

$$
I d s d \Phi=\left(\partial_{\Phi} E\right) d s d \Phi+\frac{i}{q \tau} \operatorname{Tr} P_{\tau} d P_{\tau} d P_{\tau},
$$

$$
E=\frac{1}{q} \operatorname{Tr} P_{\tau} H
$$

denotes the instantaneous energy of the system. $E(s, \Phi)$ is $2 \pi$-periodic in $\Phi$.

The first term in formula (3.13) can be interpreted as a dynamical contribution to the current (which in a QHS vanishes on the average) while the second term is of geometric origin. It almost has the form of a Chern character of a vector bundle.

Proof of Proposition 3.3. Adding zero to the right-hand side of (3.2) we get,

$$
\begin{aligned}
I= & \frac{i}{q \tau}\left\{\partial_{\Phi} \operatorname{Tr} P_{\Phi} U_{\tau}^{*} \partial_{s} U_{\tau}\right\} \\
& +\frac{i}{q \tau}\left\{\partial_{s} \operatorname{Tr} P_{\Phi} U_{\tau}^{*} \partial_{\Phi} U_{\tau}-\partial_{\Phi} \operatorname{Tr} P_{\Phi} U_{\tau}^{*} \partial_{s} U_{\tau}\right\} .
\end{aligned}
$$

Using the Schröder equation $i \partial_{s} U_{\tau}=\tau H U_{\tau}$ in the first term on the right-hand side we get

$$
I d s d \Phi=\left(\partial_{\Phi} E\right) d s d \Phi+\frac{i}{q \tau} d\left(\operatorname{Tr} P_{\Phi} U_{\tau}^{*} d U_{\tau}\right) .
$$

Now the Chern-Simons formula (3.5) can be applied to the last term on the righthand side of (3.15) with $U=U_{\tau}, P=P_{\Phi}$. This proves (3.13).

To prove periodicity of $E$ in $\Phi$, we introduce the $\Phi$-periodic Schrödinger operator

$$
\check{H}(s, \Phi)=e^{i \Phi F_{2}} H(s, \Phi) e^{-i \Phi F_{2}},
$$

where $F_{2}(x)$ was introduced in $(2.6) . \breve{H}(s, \Phi)$ is periodic in $\Phi$ by construction. Furthermore it is clear that the flow $\tilde{U}_{\tau}$ defined by the time dependent Schrödinger 
equation (2.12) with $H$ replaced by $\breve{H}$ is obtained by simply conjugating $U_{\tau}$ with the $s$-independent gauge transformation $e^{i \Phi F_{2}}$. This yields

$$
\operatorname{Tr} P_{\tau} H=\operatorname{Tr} \check{P}_{\tau} \check{H} \quad \text { with } \quad \check{P}_{\tau}=\check{U}_{\tau} \check{P}(0, \Phi) \check{U}_{\tau}^{*}
$$

and concludes the proof of Proposition 3.3.

This result gives rise to the following

Corollary 3.4. The charge transport

has the following $\Phi$-average:

$$
Q(\Phi)=\tau \int_{0}^{1} d s I(s, \Phi)
$$

$$
\langle Q\rangle=\frac{1}{2 \pi} \int_{0}^{2 \pi} d \Phi Q(\Phi)=\frac{i}{2 \pi q} \int_{R} \operatorname{Tr} P_{\tau} d P_{\tau} d P_{\tau},
$$

where $R$ denotes the rectangle $[0,1] \times[0,2 \pi]$ in $\mathbb{R}^{2}$.

Note that the energy term in (3.13) does not contribute to the average due to periodicity in $\Phi$. Unfortunately this nice formula has not been of great use for actual computations. It however strongly suggests integrality of charge transport because of its close resemblance to an integral of a Chern character over the torus.

The main advantage of formula (3.2) over formula (3.13) is the fact that it leads to an expression for the $\Phi$-averaged charge transport which is just a line integral over the boundary $s=1$ of the rectangle $R$. Formula (3.13) however produces for the charge transport the integral (3.19) over all of $R$.

\section{The Kubo Formula and its Correction Term}

This section contains our main result: In a Quantum Hall System, charge transport is quantized according to the Kubo formula up to infinite order in the adiabatic parameter $\tau$.

More precisely, we shall show

Theorem 4.1. The charge transport

$$
Q(\Phi)=\tau \int_{0}^{1} d s I(s, \Phi)
$$

has the following $\Phi$-average

$$
\langle Q\rangle=\frac{1}{2 \pi} \int_{0}^{2 \pi} d \Phi Q(\Phi)=\frac{i}{2 \pi q} \int_{T} \operatorname{Tr} \hat{P} d \hat{P} d \hat{P}+O\left(\tau^{-\infty}\right)
$$

as $\tau \uparrow \infty$.

$T$ denotes the torus $(\mathbb{R} / 2 \pi Z)^{2}$, and $\hat{P}$ is the spectral projection of rank $q$ associated to the Hamiltonian $\hat{H}$ defined in (2.8), which is a function on $T$. It thus induces a $q$-dimensional vector bundle $\hat{B}$ over $T$ with (first) Chern character

$$
\operatorname{ch} \hat{B}=i \int_{T} \operatorname{Tr} \hat{P} d \hat{P} d \hat{P} \in 2 \pi Z .
$$


Before proving the above statement we want to add a few remarks and a preparatory lemma. First of all, it is clear from (4.1) and (4.2) that charge transport in a QHS is of entirely geometric character up to infinite order in $\tau$. The right-hand side of formula (4.1) is just another way of writing the conventional Kubo formula which is well known to be of geometric character for a QHS [AS, TN].

Theorem 4.1 states that this result actually holds to all orders of perturbation theory. The main idea in the proof is to relate all correction terms to $P_{\tau}(1, \Phi)$ $-P(0, \Phi)$. This difference measures the degree to which the physical state $P_{\tau}(s, \Phi)$ "tunnels out of the initial state" $P_{\Phi}=P(0, \Phi)$ as the electric field is switched on and off again; in view of the adiabatic Theorem A4 it vanishes to infinite order in $\tau$. Finally we want to remark that we have chosen to represent the right-hand side of (4.1) in terms of the periodic projection $\hat{P}$ because it clearly defines a vector bundle over $T$ which is the basic geometric object related to charge transport. In fact, one may replace $\widehat{P}$ by any of the projections $P, \widetilde{P}, \breve{P}$ in formula (4.1).

This follows from

Lemma 4.2. Let $R=[0,1] \times[0,2 \pi]$ and let $P, \widetilde{P}, \hat{P}, \breve{P}$ denote the spectral projections associated to $H, \widetilde{H}, \widehat{H}$, and $\breve{H}$, which were introduced in Sect. II and formula (3.16) respectively. Then

$$
\int_{R} \operatorname{Tr} P d P d P=\int_{T} \operatorname{Tr} \widetilde{P} d \tilde{P} d \tilde{P}=\int_{T} \operatorname{Tr} \hat{P} d \hat{P} d \hat{P}=\int_{R} \operatorname{Tr} \check{P} d \check{P} d \check{P} .
$$

Proof. In view of definition (2.11), the equality

$$
\int_{R} \operatorname{Tr} P d P d P=\int_{T} \operatorname{Tr} \tilde{P} d \tilde{P} d \tilde{P}
$$

is a trivial consequence of the chain rule, setting $\phi_{1}=f(s)$. To prove the second equality, we recall from (2.7) and (2.8) that $\widetilde{P}$ and $\breve{P}$ are related via the unitary gauge transformation

$$
G(\phi)=\exp i\left(\phi_{1} F_{1}+\phi_{2} F_{2}\right),
$$

where $F_{j}$ are $\phi$-independent multiplication operators.

Thus the Chern-Simons formula gives

$$
\operatorname{Tr} \hat{P} d \hat{P} d \hat{P}=\operatorname{Tr} \tilde{P} d \tilde{P} d \tilde{P}+d\left(\operatorname{Tr} \widetilde{P} G^{*} d G\right) .
$$

$G^{*} d G$ is a $\phi$-independent 1 -form which commutes with $G$; thus

$$
\operatorname{Tr} \tilde{P} G^{*} d G=\operatorname{Tr} \hat{P} G^{*} d G
$$

is well defined, i.e. periodic, as a 1-form on $T$.

By Stokes theorem,

$$
\int_{T} d\left(\operatorname{Tr} \widetilde{P} G^{*} d G\right)=0 .
$$

Thus the second equality in (4.3) follows by integrating (4.5) over $T$. The last equality in (4.3) is proved analogously.

We shall now analyze the basic formula (3.2) for the Hall current by relating the physical time evolution $U_{\tau}(s, \Phi)$ to the adiabatic time evolution $U_{A}(s, \Phi)$ via the wave operator $\Omega(s, \Phi)=\left(U_{A}^{*} U_{\tau}\right)(s, \Phi)$ introduced in the Appendix. We recall that 
$U_{A}$ is the adiabatic time evolution introduced in [ASY] and defined by the initial value problem

$$
i \partial_{s} U_{A}=\tau H_{A} U_{A} ; \quad H_{A}(s, \Phi):=H(s, \Phi)+\frac{i}{\tau}\left[\partial_{s} P(s, \Phi), P(s, \Phi)\right], U_{A}\left(0, \phi_{2}\right)=1 .
$$

It intertwines spectral subspaces

$$
U_{A}(s, \Phi) P_{\Phi}=P(s, \Phi) U_{A}(s, \Phi), \quad(s, \Phi \in \mathbb{R}),
$$

and our assumption supp $\dot{f} \subset(0,1)$ ensures that

$$
\Omega(1, \Phi) P_{\Phi}=P_{\Phi} \Omega(1, \Phi) \quad \bmod O\left(\tau^{-\infty}\right),
$$

in view of Theorem A4, applied to $H(s, \Phi)$.

Furthermore, differentiability in $s$ and $\Phi$ is always settled by Lemma 2.2 since the range of the $q$-dimensional projection $P$ is contained in the $s, \Phi$-independent operator domain $D$ of $H$. With these preparations we are ready to give the

Proof of Theorem 4.1. Using $U_{\tau}=U_{A} \Omega$ we get $\bmod O\left(\tau^{-\infty}\right)$,

$$
\operatorname{Tr} P_{\Phi} U_{\tau}^{*} \partial_{\Phi} U_{\left.\tau\right|_{s=1}}=\operatorname{Tr} P_{\Phi} U_{A}^{*} \partial_{\Phi} U_{\left.A\right|_{s=1}}+\operatorname{Tr} P_{\Phi} \Omega^{*} \partial_{\Phi} \Omega_{\left.\right|_{s=1}},
$$

where we have used (4.9) and cyclicity of the trace to obtain the first term on the right-hand side. Combining (4.10) with (3.2) yields for the averaged charge transport

$$
\langle Q\rangle=\frac{i}{2 \pi q}\left\{\int_{\partial R} P_{\Phi} U_{A}^{*} d U_{A}+\int_{0}^{2 \pi} d \Phi \operatorname{Tr} P_{\Phi} \Omega^{*} \partial_{\Phi} \Omega_{\left.\right|_{s=1}}\right\} .
$$

To obtain the first term on the right-hand side of (4.11) note that

$$
\operatorname{Tr} P_{\Phi} U_{A}^{*} \partial_{s} U_{A}=\operatorname{Tr} \check{P}_{\Phi} \check{U}_{A}^{*} \partial_{s} \check{U}_{A}
$$

is periodic in $\Phi$ by use of the gauge transformation $e^{i F_{2} \Phi}$ introduced in (3.16). Since $d P_{\Phi} d P_{\Phi}=0$, the Chern-Simons formula yields

$$
\int_{\partial R} \operatorname{Tr} P_{\Phi} U_{A}^{*} \partial_{\Phi} U_{A}=\int_{R} \operatorname{Tr} P d P d P=\int_{T} \operatorname{Tr} \hat{P} d \hat{P} d \hat{P}
$$

in view of (4.8) and (4.3).

Thus it remains to show that

$$
\int_{0}^{2 \pi} d \Phi \operatorname{Tr} P_{\Phi} \Omega^{*} \partial_{\Phi} \Omega_{\mid s=1}=O\left(\tau^{-\infty}\right) .
$$

Setting $W(\Phi)=P_{\Phi} \Omega(1, \Phi) P_{\Phi}$, it follows from (4.8) that one has $\bmod 0\left(\tau^{-\infty}\right)$

$$
\begin{aligned}
\left.\operatorname{Tr} P_{\Phi} \Omega^{*} \partial_{\Phi} \Omega\right|_{s=1} & =\operatorname{Tr} W^{-1} \partial_{\Phi} W \\
& =\partial_{\Phi} \operatorname{Tr} \log W=\partial_{\Phi} \log \operatorname{det} W,
\end{aligned}
$$

where the determinant of the finite rank operator $W$ considered as a map in $\operatorname{Ran} P_{\Phi}$ is well defined. The expansion of the wave operator in Theorem A3 gives

$$
\operatorname{det} W(\Phi)=1+O\left(\tau^{-1}\right) \quad(\tau \rightarrow \infty) .
$$


Thus

$$
\omega(\Phi)=\operatorname{det} W(\Phi)=\operatorname{det} P_{\Phi} \Omega(1, \Phi) P_{\Phi}
$$

describes a closed curve in the complex plane as $\Phi$ runs from 0 to $2 \pi$ which, for $\tau$ sufficiently large, never crosses the negative real axis which serves as a branch cut in the definition of the logarithm. This proves

$$
\int_{0}^{2 \pi} d \Phi \partial_{\Phi} \log \operatorname{det} W=0
$$

and yields (4.14).

Finally we remark that the same type of argument gives by use of the intertwining property (4.8) combined with (2.8),

$$
\int_{T} \operatorname{Tr} \hat{P} d \hat{P} d \hat{P}=\int_{\partial R} \operatorname{Tr} P_{\Phi} U_{A}^{*} d U_{A}=\int_{0}^{2 \pi} d \Phi \partial_{\Phi} \log \operatorname{det} V_{A}(\Phi),
$$

where

$$
V_{A}(\Phi)=P_{\Phi} e^{2 \pi i F_{1}} U_{A}(1, \Phi) P_{\phi}
$$

is considered as a unitary operator in $\operatorname{Ran} P_{\Phi}$. curve

But now the right-hand side of (4.19) is an integer multiple of $2 \pi i$ since the

$$
[0,2 \pi] \ni \Phi \rightarrow \operatorname{det} V_{A}(\Phi)
$$

may wind around the branch point of the logarithm. Combining (4.19) and (4.13) we obtain integrality of the Chern character as claimed in (4.2). This concludes the proof of Theorem 4.1 .

\section{Appendix}

For completeness sake we collect in this appendix the main points about the adiabatic evolution of time dependent Schrödinger operators explained in [ASY]. The main point is to correct an error in the proof of Lemma 2.7 of that reference. It states that for Hamiltonians with energy bands separated by gaps, tunneling out of such energy bands decays faster than any inverse power of the adiabatic time scale $\tau$. This is crucial for our analysis of the corrections to quantized charge transport in Sect. IV.

Let $\mathscr{H}$ be a Hilbert space and denote by $I$ a bounded open interval containing zero. We consider time dependent Hamiltonians satisfying:

(i) For $s \in I H(s)$ is a family of operators on $\mathscr{H}$, which is self-adjoint on some $s$-independent domain $D \subset \mathscr{H}$ and bounded from below.

(ii) Topologizing $D$ with the graph norm of $H(0)$, the function $H(s)$ is $C^{k}$ as a map from $I$ to the Banach space $B(D, \mathscr{H})$ of bounded linear operators.

(iii) $H(s)$ has gaps in its spectrum and $P(s)$ is the spectral projection on a finite band bordered by gaps; i.e. there are two continuous functions $g_{ \pm}: I \rightarrow \mathbb{R}$ and $\varepsilon>0$ such that

$$
\operatorname{dist}\left[\left\{g_{+}(s), g(s)\right\} ; \sigma(H(s))\right]>\varepsilon, \quad(s \in I) .
$$


It follows from (i) and (ii) that the resolvent $R(s, z)=[H(s)-z]^{-1}$ is $C^{k}$ as a map from $I$ to the space $B(\mathscr{H})$ of bounded linear operators on $\mathscr{H}$. The same is true for the projection

$$
P(s)=\frac{1}{2 \pi i} \int_{\Gamma} R(s, z) d z,
$$

where $\Gamma$ denotes a contour encircling the band of the spectrum associated with $P(s)$ in the clockwise sense.

For brevity we henceforth freely suppress dependence on $s \in I$ in $H, R(z), P$ and use the notation $Q=1-P, P_{0}=P(0)$.

Defining the adiabatic operator associated to $H$ and $P$ with time scale $\tau$ by

$$
H_{A}=H_{A}(s, P)=H+\frac{i}{\tau}[\dot{P}, P],
$$

the theorem of Kato and Yoshida on existence and uniqueness of the solution to the time dependent Schrödinger equation can be applied to $H$ and $H_{A}$, yielding

Theorem A1. Let $k \geqq 2, \tau>0$. Then, for all $\Psi \in D$, the initial value problems

$$
\begin{array}{rlrl}
i \partial_{s} U_{\tau} \Psi & =\tau H U_{\tau} \Psi ; & U_{\tau}(0) \Psi=\Psi ; \\
i \partial_{s} U_{A} \Psi=\tau H_{A} U_{\tau} \Psi ; & U_{A}(0) \Psi=\Psi ;
\end{array}
$$

have a unique solution which satisfies:

$U_{\tau}(s), U_{A}(s)$ are unitary, strongly continuous in $s \in I$ and $U_{\tau} \Psi, U_{A} \Psi \in C^{1}(I, \mathscr{H})$ for all $\Psi \in D$.

It then follows readily from Eq. (A4) that the adiabatic evolution $U_{A}=U_{A}(s, P)$ intertwines the spectral subspaces of $H$ in the sense that

$$
U_{A}(s, P) P_{0}=P(s) U_{A}(s, P) \quad(s \in I) .
$$

To compare the adiabatic with the physical evolution one considers the "wave operator"

$$
\Omega(s)=U_{A}^{*}(s, P) U_{\tau}(s) \quad(s \in I) .
$$

Introducing the kernel

$$
K=K_{\tau}(s, P)=U_{A}^{*}[\dot{P}, P] U_{A},
$$

it readily follows from (A 3 ) and (A4) that $\Omega$ satisfies the Volterra integral equation

$$
\Omega(t)=1-\int_{0}^{t} K_{\tau}(s, P) \Omega(s) d s \quad(t \in I) .
$$

Since $K_{\tau}(s, P)$ is a (strongly) continuous kernel for $s \in I$, the standard iteration procedure

$$
\Omega_{0}(t)=1, \quad \Omega_{j}(t)=-\int_{0}^{t} K_{\tau}(s, P) \Omega_{j-1}(s) d s \quad(j \geqq 1)
$$

yields a convergent expansion for $\Omega(t)$.

However, to make this into an asymptotic expansion with respect to the adiabatic time scale $\tau$, the rapid oscillations in $U_{A}(t, P)$ have to be used. 
To prove such a result, one must combine the specific algebraic properties of $[\dot{P}, P]$ and the intertwining property (A5) with the following formula for partial integration:

Lemma A2. Suppose $H$ satisfies (i-iii) above with $k \geqq 2$. Let $X, Y: I \rightarrow B(\mathscr{H})$ be strongly $C^{1}$. For all $t \in I$, let

$$
\tilde{X}(t)=-\frac{1}{2 \pi i} \int_{\Gamma} R(t, z) X(t) R(t, z) d z .
$$

Then

$$
\begin{aligned}
\int_{0}^{t} Q_{0} & \left(U_{A}^{*} X U_{A}\right)(s) P_{0} Y(s) d s \\
= & \frac{i}{\tau} Q_{0}\left\{\left.\left(U_{A}^{*} \tilde{X} U_{A}\right)(s) P_{0} Y(s)\right|_{0} ^{t}-\int_{0}^{t}\left(U_{A} \tilde{X} U_{A}^{*}\right)(s) P_{0} \dot{Y}(s) d s\right\} \\
& -\int_{0}^{t} U_{A}^{*}(s)(\dot{\tilde{X}}+[\dot{P}, \tilde{X}])(s) U_{A}(s) P_{0} Y(s) d s .
\end{aligned}
$$

Furthermore, if $P$ and $Q$ are interchanged in formula(A11), the right-hand side has to be multiplied by $(-1)$.

For the proof of Lemma A2 we refer to [ASY]. To understand the meaning of interchanging $P$ and $Q$, one simply observes that the right-hand side of (A11) is linear in $\widetilde{X}$ which depends on the orientation of the contour $\Gamma$ enclosing the band spectrum of $H$ associated with $P$. As a curve on the Riemann sphere, $\Gamma$ then encloses the spectrum of $H$ associated with $Q=1-P$ with opposite orientation; thus interchanging $P$ and $Q$ introduces a factor of $(-1)$ in the twiddle operation.

Lemma A 2 serves to exhibit the asymptotic nature of the expansion (A9) with respect to $\tau$ :

Theorem A3. Suppose $H$ satisfies (i-iii) above with $k \geqq 2$.

Let

$$
R_{N}(s)=\Omega(s)-\sum_{j=0}^{N} \Omega_{j}(s)
$$

Then

$$
\begin{array}{ll}
\left\|\Omega_{2 j-1}(s)\right\|+\left\|\Omega_{2 j}(s)\right\|=O\left(\tau^{-j}\right) & (j \geqq 1), \\
\left\|R_{2 j}(s)\right\|+\left\|R_{2 j+1}(s)\right\|=O\left(\tau^{-j-1}\right) & (j \in \mathbb{N})
\end{array}
$$

locally uniformly in $s \in I$.

Proof. The proof of Theorem 2.4 in [ASY] yields by use of Lemma A2,

$$
\left\|\Omega_{n+1}(s)\right\| \leqq C / \tau \sup _{0<t<s}\left(\left\|\Omega_{n}(t)\right\|,\left\|\Omega_{n-1}(t)\right\|\right)
$$

locally uniformly in $s \in I$. Since $\Omega_{0}=1$ and $\Omega_{1}=O\left(\tau^{-1}\right)$, one gets (A12) by induction on $n$. Using the integral equation for the remainder term,

$$
R_{N}(s)=-\int_{0}^{s} K_{\tau}(t) R_{N-1}(t) d t,
$$


one derives as above the estimate (A14) with $\Omega_{n}$ replaced by $R_{n}$. The crude bounds $R_{0}=O(1), R_{1}=O\left(\tau^{-1}\right)$ first yield (A12) with $\Omega_{n}$ replaced by $R_{n}$. Equation (A13) then follows from

by use of A12.

$$
R_{n}=\Omega_{n+1}+R_{n+1}
$$

Next we come to the main point of this appendix: If the time dependence of $H(s)$ is smoothly switched on and off, then tunneling out of spectral subspaces is small.

Theorem A4. Let $H$ satisfy (i-iii) above for all $k \in \mathbb{N}$, and suppose $\dot{H}$ to be compactly supported within $(0,1) \subset I$. Then

$$
\left\|P_{0} \Omega(t) Q_{0}\right\|+\left\|Q_{0} \Omega(t) P_{0}\right\|=O\left(\tau^{-\infty}\right)
$$

uniformly in $t \in I \backslash(0,1)$.

The essential idea in proving (A14) is to repeatedly apply Lemma A2 to $\Omega_{j}(s)$ with $X=[\dot{P}, P]$,

$$
Y(s)=\int_{0}^{s} d s_{1} K\left(s_{1}\right) \int_{0}^{s_{1}} d s_{2} \ldots \int_{0}^{s_{k-1}} d s_{k} K\left(s_{k}\right)
$$

for some $k \in \mathbb{N}$, gaining a factor $\tau^{-1}$ at each step.

Note, however, that the higher derivatives $\partial^{j} Y(s)$ for $j \geqq 2$ involve derivatives of $U_{A}(s)$ up to order $j-1$, and these will grow like $\tau^{j-1}$. In view of the intertwining property (A5) we will, however, explicitly show that no derivatives of $U_{A}(s)$ occur upon partial integration in the formula for $\Omega_{j}(s)$. To this end we introduce some more notation and prove a technical lemma which then will yield the proof of Theorem A4.

We set $M=C_{0}^{\infty}((0,1), B(\mathscr{H}))$ where differentiability is understood in the norm sense. For $X_{j}, Z_{j} \in M\left(j \in \mathbb{N}^{*}\right)$ we set

and define for all $n \in \mathbb{N}$

$$
F_{j}=U_{A}^{*} X_{j} U_{A}, \quad G_{j}=U_{A}^{*} Z_{j} U_{A}
$$

$$
A_{n}^{0}:=A_{n}^{0}\left(X_{1}, \ldots, X_{2 n+1}\right)=P_{0} \int_{0}^{1} d t_{1} F_{1}\left(t_{1}\right) Q_{0} \int_{0}^{t_{1}} d t_{2} \ldots P_{0} \int_{0}^{t_{2 n}} d t_{2 n+1} F_{2 n+1}\left(t_{2 n+1}\right) Q_{0}
$$

(we set $t_{0}=1$ ).

Next we define $A_{n}^{l}\left(X_{1}, \ldots, X_{2 n+1}\right)$ for $1 \leqq l \leqq n$ by replacing $Q_{0}$ by $P_{0}$ on the right-hand side of (A19), after $F_{1}, F_{3}$ up to $F_{2 l-1}$. Similarly, we set

$$
B_{n}^{0}:=B_{n}^{0}\left(Z_{1}, \ldots, Z_{2 n+2}\right)=P_{0} \int_{0}^{1} G_{1} P_{0} \int_{0}^{t_{1}} G_{2} Q_{0} \ldots P_{0} \int_{0}^{t_{2 n+1}} G_{2 n+2} Q_{0}
$$

in an obvious shorthand and define $B_{n}^{l}\left(Z_{1}, \ldots, Z_{2 n+2}\right)$ for $1 \leqq l \leqq n$ by replacing $Q_{0}$ by $P_{0}$ on the right-hand side of (A20), after $G_{2}, G_{4}$ up to $G_{2 l}$. Furthermore we set

We claim

$$
A_{n}^{l}=0=B_{n}^{l} \quad(l \in \mathbb{Z} \backslash\{0, \ldots, n\}) .
$$

Lemma A5. With the hypothesis of Theorem A4, we have

$$
\begin{gathered}
\forall n, l \in \mathbb{N}, \quad \forall X_{1}, \ldots, X_{2 n+1}, Z_{1}, \ldots, Z_{2 n+2} \in M: \\
\left\|A_{n}^{l}\left(X_{1}, \ldots, X_{2 n+1}\right)\right\|+\left\|B_{n}^{l}\left(Z_{1}, \ldots, Z_{2 n+2}\right)\right\|=O\left(\tau^{-\infty}\right) .
\end{gathered}
$$


Furthermore, if $P$ and $Q$ are interchanged in the definition of $A_{n}^{l}$ and $B_{n}^{l}$, then (A22) still holds true.

Proof. The proof is by induction on $n$. In each step we use induction again. For brevity we suppress the quantifier: $\forall X_{j}, Z_{i} \in M$.

For $n=0$ we have to show:

$$
\forall N \in \mathbb{N} \quad\left\|A_{0}^{0}\left(X_{1}\right)\right\|+\left\|B_{0}^{0}\left(Z_{1}, Z_{2}\right)\right\|=O\left(\tau^{-N}\right) .
$$

This is obviously true for $N=0$. Furthermore, it follows from Lemma A2 that

$$
A_{0}^{0}(X)=\frac{i}{\tau} A_{0}^{0}(\dot{\tilde{X}}+[\dot{P}, \tilde{X}]),
$$

and, in view of the intertwining property, that

$$
B_{0}^{0}\left(Z_{1}, Z_{2}\right)=-\frac{i}{\tau}\left\{A_{0}^{0}\left(Z_{1} P \tilde{Z}_{2}\right)-B_{0}^{0}\left(Z_{1}, \dot{\tilde{Z}}_{2}+\left[\dot{P}, \widetilde{Z}_{2}\right]\right)\right\} .
$$

Our hypothesis implies that $P \in C^{\infty}(I, B(\mathscr{H}))$ and $\dot{P} \in M$. Clearly $M$ is stable under differentiation, the twiddle operation and the operation of taking commutators; furthermore $P Z \in M$ for all $Z \in M$. Thus (*) follows by induction on $N$ from (A23), (A24).

Next we come to the induction step with respect to $n$. Assuming (A22) for (fixed) $n-1$, we have to show

$$
\begin{gathered}
\left\|A_{n}^{l}\left(X_{1}, \ldots, X_{2 n+1}\right)\right\|+\left\|B_{n}^{l}\left(Z_{1}, \ldots, Z_{2 n+2}\right)\right\|=O\left(\tau^{-N}\right) \\
\left(N, l \in \mathbb{N}, X_{1}, \ldots, X_{2 n+1}, Z_{1}, \ldots, Z_{2 n+2} \in M\right) .
\end{gathered}
$$

The proof is by induction on $N$. For $N=0(* *)$ is obviously true. Assume $0 \leqq l \leqq n$. Setting $X=X_{2 l+1}$,

$$
Y\left(t_{2 l+1}\right)=\int_{0}^{t_{2 l+1}} K_{2 l+2} P_{0} \ldots \int_{0}^{t_{2 n}} P_{0} K_{2 n+1} Q_{0} \quad(1<n)
$$

(respectively zero for $1=n$ ) we apply Lemma A 2 to the term

$$
\int_{0}^{t_{2 l}} P_{0}\left(U_{A}^{*} X U_{A}\right)(s) Q_{0} Y(s) d s
$$

in the definition (A17) of $A_{n}^{l}$ and obtain

$$
\begin{aligned}
A_{n}^{l}\left(X_{1}, \ldots, X_{2 n+1}\right)= & -\frac{i}{\tau}\left\{B_{n-1}^{l-1}\left(X_{1}, \ldots, X_{2 l-1}, X_{2 l} P \tilde{X}, X_{2 l+2} \ldots\right)\right. \\
& -B_{n-1}^{l}\left(X_{1}, \ldots, X_{2 l}, \tilde{X} Q X_{2 l+2}, X_{2 l+3}, \ldots\right) \\
& \left.-A_{n}^{l}\left(X_{1}, \ldots, X_{2 l}, \tilde{\tilde{X}}+[\dot{P}, \tilde{X}], X_{2 l+2}, \ldots\right)\right\} .
\end{aligned}
$$

Similarly we set $Z=Z_{2 l+2}$ and integrate by parts in the definition of $B_{n}^{l}$ to get

$$
\begin{aligned}
B_{n}^{l}\left(Z_{1}, \ldots, Z_{2 n+2}\right)= & -\frac{i}{\tau}\left\{A_{n}^{l}\left(Z_{1}, \ldots, Z_{2 l}, Z_{2 l+1} P \tilde{Z}, Z_{2 l+3}, \ldots\right)\right. \\
& -A_{n}^{l+1}\left(Z_{1}, \ldots, Z_{2 l+1}, \tilde{Z} Q Z_{2 l+3}, Z_{2 l+4}, \ldots\right) \\
& \left.-B_{n}^{l}\left(Z_{1}, \ldots, Z_{2 l+1}, \tilde{\tilde{Z}}+[\dot{P}, \tilde{Z}], Z_{2 l+3}, \ldots\right)\right\} .
\end{aligned}
$$


Thus (**) follows by induction on $N$ from (A25), (A26), where we use the induction hypothesis (A22) for fixed (n-1) to estimate the right-hand side of (A25). This proves (A22). Finally, interchanging $P$ and $Q$ in the definition of $A_{n}^{l}$ and $B_{n}^{l}$ just multiplies the right-hand side of (A23)-(A26) by (-1). This proves the lemma.

Now we are ready for the

Proof of Theorem A4. In view of Theorem A3, it suffices to prove (A14) with $\Omega$ replaced by $\Omega_{j}, j \in \mathbb{N}$. Since $P \dot{P} P=0$, it follows readily from (A5) that

$$
P_{0} K P_{0}=Q_{0} K Q_{0}=0 .
$$

By definition of $\Omega_{j}$, this implies inductively for all $j \in \mathbb{N}$,

$$
P_{0} \Omega_{2 j} Q_{0}=Q_{0} \Omega_{2 j} P_{0}=0
$$

and

$$
P_{0} \Omega_{2 j+1} Q_{0}=-A_{j}^{0}([\dot{P}, P], \ldots,[\dot{P}, P]) .
$$

Since $[\dot{P}, P] \in M,(\mathrm{~A} 16)$ follows from Lemma A 5 .

Acknowledgements. This work has been supported by the Stiftung Volkswagenwerk and the Deutsche Forschungsgemeinschaft. It was in part done during a stay at the Swiss Federal Institute of Technology in Zürich. We thank in particular Jürg Fröhlich and Walter Hunziker for their kind hospitality. To both of them and R. Gerhardts and R. J. Haug at the MPI Stuttgart we are grateful for interesting discussions.

\section{References}

[AS] Avron, J.E., Seiler, R.: Quantisation of the Hall conductance for general multiparticle Schrödinger Hamiltonians. Phys. Rev. Lett. 54, 259-262 (1985)

[ASS] Avron, J.E., Seiler, R., Shapiro, B.: Generic properties of quantum Hall Hamiltonians for finite systems. Nucl. Phys. B 265, 364-374 (1986)

[ASY] Avron, J.E., Seiler, R., Yaffe, L.G.: Adiabatic theorems and applications to the quantum Hall effect. Commun. Math. Phys. 110, 33-49 (1987)

[K1] Kato, T.: Perturbation theory for linear operators. Berlin, Heidelberg, New York: Springer 1984

[K2] Kato, T.: On the adiabatic theorem of quantum mechanics. J. Phys. Soc. J. Jpn. 5, 435-439 (1950)

[Kr] Krein, S.G.: Linear differential equations in Banach space. Transl. Math. Mon. 27 (1972)

[L] Laughlin, R.B.: Quantized Hall conductivity in two dimensions. Phys. Rev. B 23, 5632-5633 (1981)

$\left[\mathrm{TKN}^{2}\right]$ Thouless, D.J., Kohmoto, M., Nightingale, M., den-Nys, M.: Quantized Hall conductance in a two dimensional periodic potential. Phys. Rev. Lett. 49, 405-408 (1982)

[TN1] Thouless, D.J., Niu, Q.: Nonlinear corrections to the quantization of Hall conductance. Phys. Rev. B 30, 3561-3562 (1984)

[TN2] Thouless, D.J., Niu, Q.: Quantised adiabatic charge transport in the presence of substrate disorder and many-body interaction. J. Phys. A 17, 2453-2462 (1984)

[Y] Yoshida, K.: Functional analysis. Grundlehren der math. Wiss. Bd. 123, Berlin, Heidelberg, New York: Springer

Communicated by B. Simon

Received March 8, 1989; in revised form June 13, 1989 